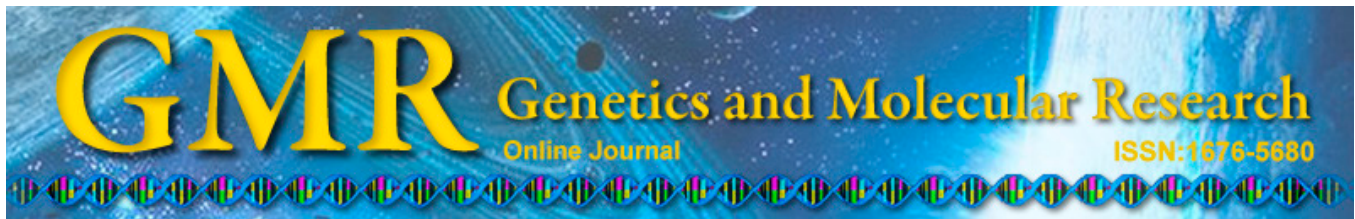

\title{
Roles of the AIB1 protein in the proliferation and transformation of human esophageal squamous cell carcinoma
}

\author{
L. Li ${ }^{1 *}$, P. Wei ${ }^{2 *}$, M.-H. Zhang ${ }^{1}$, W. Zhang ${ }^{2}$, Y. Ma ${ }^{1}$, X. Fang ${ }^{1}$, C.-L. Hao ${ }^{1}$ \\ and Z.-H. Zhang ${ }^{3}$ \\ ${ }^{1}$ The Third Department of Oncology, People's Hospital of Tengzhou City, \\ Shandong, China \\ ${ }^{2}$ Department of Oncology, The Tumor Hospital of Shandong Province, Jinan, \\ Shandong, China \\ ${ }^{3}$ Shandong Occupational Health and Occupational Prevention Institute, Jinan, \\ Shandong, China \\ *These authors contributed equally to this study. \\ Corresponding author: L. Li \\ E-mail: liling_tz@163.com
}

Genet. Mol. Res. 14 (3): 10376-10383 (2015)

Received November 12, 2014

Accepted March 18, 2015

Published September 1, 2015

DOI http://dx.doi.org/10.4238/2015.September.1.4

\begin{abstract}
The aim of this study was to investigate the expression of AIB1 in human esophageal squamous cell carcinoma and its correlation with Ki67 expression. The immunohistochemical method streptavidinperosidase was used to analyze the expression of AIB1 and $\mathrm{Ki} 67$ in specimens from 60 patients with esophageal squamous cell carcinoma and in 20 control individuals with normal esophageal tissue. Expression correlation, clinical significance, and relationships between the two groups were determined. In the 20 individuals with normal esophageal mucosa cells, AIB expression was primarily detected at low levels in the nucleus or not at all, whereas $41.6 \%$ of specimens from individuals with esophageal squamous cell carcinoma exhibited high levels of
\end{abstract}


AIB1 expression $(\mathrm{P}<0.05)$. Furthermore, overexpression of AIB1 was observed more frequently in carcinoma specimens with late T stages (T3/ T4) and lymph node metastases $(\mathrm{P}<0.05)$. No significant differences were observed in AIB1 expression by gender, age, or pathological type $(\mathrm{P}<0.05)$. Comparatively, the rate of positive expression of Ki67 In esophageal squamous cell carcinoma specimens was $65.0 \%(39 / 60)$ $(\mathrm{P}<0.05)$. Of these, 29 specimens exhibited simultaneous expression of AIB1, 25 of which demonstrated AIB1 overexpression; expression of AIB1 and Ki67 was positively correlated $(\mathrm{P}<0.01)$. In summary, the results from this study suggested that AIB1 protein expression was associated with the $\mathrm{T}$ stage and lymph node metastasis in esophageal squamous cell carcinoma, and that Ki67 might play a role in the AIB1 non-steroid receptor pathway.

Key words: Esophageal; Squamous cell carcinoma; AIB1; Ki67

\section{INTRODUCTION}

AIB1 is a member of the steroid receptor co-activator (SRC) family (Lahusen et al., 2009), and has been shown to be over-expressed in breast cancer, ovarian cancer, and in other hormone-dependent tumors (Alkner et al., 2010; Torres-Arzayus et al., 2010); however, few studies have examined the expression of AIB1 in non-hormone-dependent tumors such as esophageal carcinoma. The primary mechanism of AIB1 function is via the steroid hormone receptor pathway, wherein AIB1 interacts with estrogen receptors to promote cell proliferation and tumor formation (Zhao et al., 2009). Furthermore, recent studies have demonstrated that AIB1 also functions via the non-steroid hormone receptor pathway; therefore, AIB1 might affect the cell cycle and cell proliferation through this mechanism as well, and in addition, might interact with apoptosis genes such as $p 53, B C L 2$, and others, thus affecting tumor development and progression (Lee et al., 1999; Han et al., 2008; Yamashita er al., 2009; Ma et al., 2011). The Ki67 protein is also related to cell division and proliferation. It serves as a positive marker gene for cell proliferation, and its expression has been shown to be related to the occurrence, development, metastasis, and prognosis of various tumors (Gentile et al., 2006; Jalava et al., 2006; Sun et al., 2008a). It remains to be verified whether Ki67 is related to the AIB1 nonsteroidal hormone receptor pathway. In this study, in order to study the expression of AIB1 in esophageal cancer and its correlation with Ki67 expression, we used immunohistochemical methods to detect the expression of AIB1 and of the proliferating cell nuclear antigen Ki67 in normal esophageal epithelium and in esophageal squamous cell carcinoma specimens, and explored the clinical relevance thereof. We expect that the results from this study will provide new ideas for future research.

\section{MATERIAL AND METHODS}

\section{Material}

For this study, 80 specimens were collected from Tengzhou Central People's Hospital between January 12, 2008 and December 23, 2010, comprising 60 specimens from pa- 
tients with esophageal squamous cell carcinoma and 20 specimens from individuals which also come from above patients with normal esophageal tissue. Of the esophageal squamous cell carcinoma specimens, 47 were from men and 13 from women; patients were aged 42-79 years, with an average age of $(64 \pm 12)$ years and median age of 64.2 years, while of the normal esophageal tissue, 12 were from men and 8 from women; patients were aged $40-75$ years, with ., with an average age of $(61 \pm 10)$ years and median age of 61.5 years. The characteristics of the carcinoma specimens were as follows: differentiation grade: I-II (27 specimens), II-III (33 specimens); infiltration degree [tumor, node, metastasis (TNM) staging]: T1-T2 (24 specimens), T3-T4 (36 specimens); and 28 and 32 patients did/did not exhibit lymph node metastasis. No patient received chemotherapy treatment. Diagnosis was confirmed through examination of paraffin specimens by our hospital pathologist. The study was approved by the relevant institutional ethical oversight committee for clinical studies. All subjects provided signed informed consent.

\section{Main reagents}

Mouse-anti-human AIB1 monoclonal antibody and rabbit-anti-human proliferating cell nuclear antigen (Ki67) monoclonal antibody were purchased from Shanghai University Biotechnology Co., Ltd. and Shanghai Sun Bio-Technique Co. Ltd. (Shanghai, China), respectively; the immunohistochemistry kit was purchased from ZSGB Bio-Technique Co. Ltd. (Beijing, China).

\section{Methods}

Sections of normal esophageal tissue, tumor-adjacent tissue (defined as over $3 \mathrm{~cm}$ distant from esophageal squamous cell carcinoma tissue) and esophageal squamous cell carcinoma were prepared according to the manufacturer protocol. Paraffin-embedded specimens were fixed with $10 \%$ formalin. Serial sections $(4 \mu \mathrm{m})$ were baked in a $60^{\circ} \mathrm{C}$ oven for $2 \mathrm{~h}$, Sections were washed with phosphate buffered saline (PBS), and subjected to high-pressure hot fixes: citrate buffer was added to a pressure cooker, and sections were added after boiling for $2 \mathrm{~min}$ and allowed to cool naturally. Following washing with PBS, sections were added to 3\% $\mathrm{H}_{2} \mathrm{O}_{2}$ to react at room temperature for $10 \mathrm{~min}$, and washed again. Goat serum which can effectively block tissue nonspecific sites combining with antibody was added at room temperature and blocking for $10 \mathrm{~min}$, followed by removal of the serum, addition of mouse anti-human AIB1 monoclonal antibody at a dilution of 1:100, and then addition of rabbit-anti-human Ki67 monoclonal antibody at a dilution of 1:50. Primary antibodies were allowed to bind at room temperature for $2 \mathrm{~h}$, followed by a PBS wash and then addition of secondary antibody working solution with incubation at room temperature for $1 \mathrm{~h}$. After another PBS wash, streptavidinperoxidase was added at room temperature and allowed to react for $20 \mathrm{~min}$, with a final PBS wash. Sections were stained for 4 min with a 3,3' diaminobenzidine (DAB) dye solution, washed with water, stained with hematoxylin for $30 \mathrm{~s}$, and placed into PBS buffer to return to visualize the blue stain. Finally, sections were dehydrated with a graded ethanol series (85, 95, and absolute I and II) and xylene solutions I and II for 5 min each, and blocked with gum which can avoid air oxidation of sections. 


\section{Evaluation standards}

Specimens were graded by two pathologist who are visiting staff according to the size and color of the stained sample. Yellow granules in the cytoplasm and nucleus represented AIB1 positive expression. Brown granules in the nucleus represented Ki67 positive expression. Five fields were randomly selected under the microscope (400X magnification) and 100 cells were scored per field. For AIB1, specimens were scored and classified according to the percentage of positive cells as follows: $(-)$ represented $<5 \%$; $(+)$ represented $5-50 \%$; $(++)$ represented $>50 \%$; and $(+++)$ equaled approximately $100 \%$. For Ki67, specimens were graded according to the proportion of the number of nuclei colored in 200 squamous epithelium cells per field from five randomly selected fields, with the mean percentage of positive cells scored as follows: (-) represented 0-5\%; (+) represented 5-25\%; (++) represented 26-50\%; (+++) represented $50-75 \%$; and $(++++)$ represented $>75 \%$.

\section{Statistical analysis}

Data were analyzed using SPSS 16.0 (SPSS, Chicago, IL, USA). Ranked data were analyzed using a rank sum test. Comparisons were performed using a $\chi^{2}$ test among groups; the correlation of AIB1 and Ki67 expression was performed using categorical data. Testing standard: $\alpha=0.05 . \mathrm{P}<0.05$.

\section{RESULTS}

\section{AIB1 and Ki67 expression in esophageal squamous cell carcinoma and normal esophageal tissues}

The highest expression of AIB1 protein detected in normal esophageal tissue and tumor-adjacent tissue was represented as "+", so therefore "++" - "+++" was defined as AIB1 protein over-expression. As can be seen from Figure 1A-D, AIB1 and Ki67 expression was localized in the nucleus. Of the 60 specimens from patients with esophageal squamous cell carcinoma, 25 (41.6\%) exhibited AIB1 over-expression, compared to the weak or lack of expression observed in the 20 specimens of normal esophageal tissue. (Figure 1B). Therefore, overall, the AIB1 expression in esophageal squamous cell carcinoma was significantly higher than that in normal esophageal squamous epithelium tissue $(\mathrm{P}<0.05)$. In addition, the percentage of specimens positive for Ki67 protein expression in normal esophageal tissues and tumor-adjacent tissues was only 15\%, far below the percentage of specimens positive for Ki67 protein expression $(65 \%)$ in esophageal squamous cell carcinoma $(\mathrm{P}<0.05)$.

\section{Relationship between AIB1 expression and clinical pathology of esophageal squa- mous cell carcinoma}

AIB1 expression was significantly correlated with clinical stage and lymph node metastasis $(\mathrm{P}<0.05)$, but it was not associated with gender, age, or tumor grade. Comparatively, the positive expression of Ki67 protein in esophageal squamous cell carcinoma tissue in T3-4 stage was higher than T1-2 stage, and was also significantly correlated with differentiation degree. Poorly differentiated carcinoma tissue exhibited high levels of Ki67 expression, and this 
correlated with lymph node metastasis $(\mathrm{P}<0.05)$, but not with gender or age $(\mathrm{P}>0.05)($ Table 1$)$.

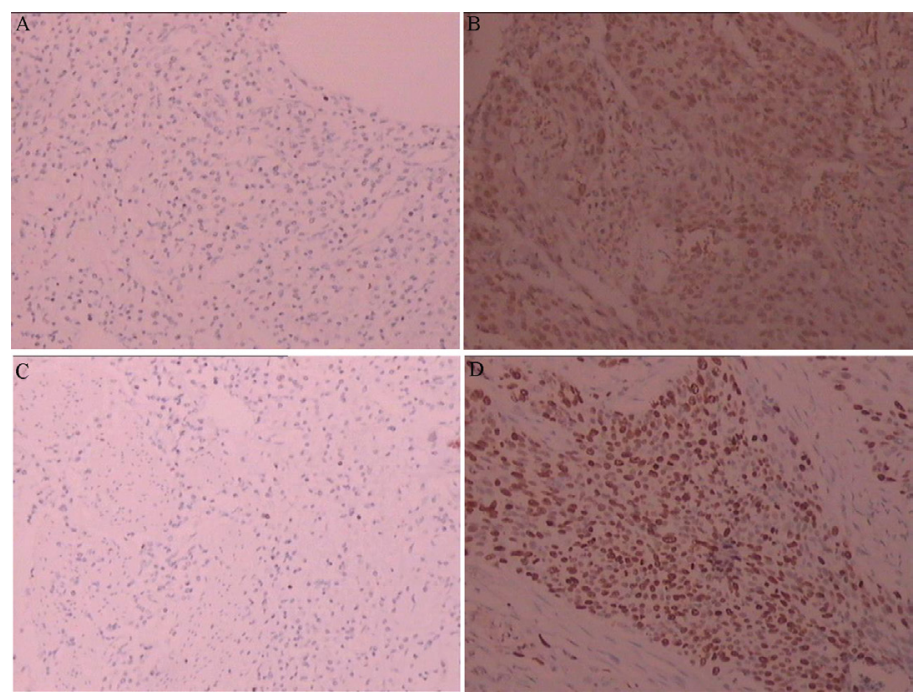

Figure 1. Immunohistochemical analysis of AIB1 and Ki67 expression in normal esophageal tissues and esophageal squamous cell carcinoma. A. Lack of expression AIB1 pyrenoid in the normal esophageal tissue; $\mathbf{B}$. AIB1 pyrenoid over-expression in esophageal squamous cell carcinoma; C. lack of expression Ki67 pyrenoid in the normal esophageal tissue; D. Ki67 pyrenoid over-expression in esophageal squamous cell carcinoma.

Table 1. Correlation of clinical data with AIB1 and Ki67 expression.

\begin{tabular}{|c|c|c|c|c|c|c|c|}
\hline \multirow[b]{2}{*}{ Observation Indexes } & \multirow[b]{2}{*}{$\mathrm{N}$} & \multicolumn{3}{|c|}{ AIBI } & \multicolumn{3}{|c|}{ Ki67 } \\
\hline & & - & + & $\mathrm{P}$ & - & + & $\mathrm{P}$ \\
\hline \multicolumn{8}{|l|}{ Age (years) } \\
\hline$\geq 64$ & 35 & $20(57.1)$ & $15(42.9)$ & \multirow[t]{2}{*}{0.315} & $11(31.4)$ & $24(68.6)$ & \multirow[t]{2}{*}{0.49} \\
\hline$<64$ & 25 & $11(44.0)$ & $14(56.0)$ & & $10(40.0)$ & $15(60.0)$ & \\
\hline \multicolumn{8}{|l|}{ Gender } \\
\hline Male & 37 & $18(48.6)$ & $19(51.4)$ & \multirow[t]{2}{*}{0.553} & $13(35.1)$ & $24(64.9)$ & \multirow[t]{2}{*}{0.978} \\
\hline Female & 23 & $13(56.5)$ & $10(43.5)$ & & $8(34.8)$ & $15(65.2)$ & \\
\hline \multicolumn{8}{|c|}{ Lymph node metastasis } \\
\hline No & 28 & $9(32.1)$ & $19(67.9)$ & \multirow[t]{2}{*}{0.005} & $6(21.43)$ & $22(78.57)$ & \multirow[t]{2}{*}{0.035} \\
\hline Yes & 32 & $22(68.8)$ & $10(31.3)$ & & $15(46.9)$ & $17(53.1)$ & \\
\hline \multicolumn{8}{|l|}{$\mathrm{T}$ staging } \\
\hline $\mathrm{T} 1-2$ & 24 & $17(70.8)$ & $7(29.2)$ & \multirow[t]{2}{*}{0.009} & $13(54.2)$ & $11(45.8)$ & \multirow[t]{2}{*}{0.011} \\
\hline $\mathrm{T} 3-4$ & 36 & $14(38.9)$ & $22(61.1)$ & & $8(22.2)$ & $28(77.8)$ & \\
\hline \multicolumn{8}{|l|}{ Differentiation } \\
\hline Mild & 14 & $8(57.1)$ & $6(42.9)$ & \multirow{3}{*}{0.753} & $0(0)$ & $14(100)$ & \multirow{3}{*}{0.007} \\
\hline Moderate & 24 & $11(45.8)$ & $13(54.2)$ & & $11(45.8)$ & $13(54.2)$ & \\
\hline Severe & 22 & $12(54.5)$ & $10(45.5)$ & & $10(50.0)$ & $12(50.0)$ & \\
\hline
\end{tabular}

\section{Correlation analysis of AIB1 and Ki67 expression}

Of the 39 esophageal squamous cell carcinoma specimens positive for Ki67 protein expression, 29 had simultaneous AIB1 expression, including 25 with AIB1 over-expression. Statistical analysis by correlation of categorical data indicated that AIB1 and Ki67 protein expression showed moderate positive correlation $(\mathrm{P}<0.01)$. Accordingly, of the 29 specimens 
that demonstrated AIB1 protein expression, all were positive for Ki67 expression. However, in 31 esophageal squamous cell carcinoma specimens of AIB1 protein negative expression, only 10 cases had simultaneously Ki67 positive expression. Correlation analysis also indicated that AIB1 and Ki67 protein expression showed moderate positive correlation $(\mathrm{P}<0.01)$.

\section{DISCUSSION}

The $A I B 1$ gene, also known as $S R C-3$, encodes a transcriptional co-activator of steroid hormone receptors, and is a member of the P160 family of nuclear hormone receptor co-activators (Kirkegaard et al., 2007). The AIB1 gene was first identified in human breast cancer and it is located on chromosome 20q12. AIB1 contains 1420 amino acids with a relative molecular mass of $156 \times 10^{3} \mathrm{kDa}$ (Kuang et al., 2008). Many studies have focused on the role of AIB1 in hormone-dependent tumors, demonstrating that AIB1 is over-expressed in breast and ovarian cancers, and in other hormone-dependent tumor tissues. The primary mechanism of AIB1 function in these tumors is through the steroid hormone receptor pathway; namely, the AIB1 protein interacts with estrogen receptors, and can strongly enhance their promotion of target gene transcription, thereby causing cell proliferation and tumor formation (Anzick et al., 1997), thus affecting the malignant phenotypes of hormone-dependent tumors. In recent years, however, a growing number of reports have indicated that abnormal AIB1 expression could also be identified in liver, stomach, colon, and pancreatic cancers as well as in other non-hormone-dependent tumors. Together, these results indicate that the mechanisms of AIB1 function include both steroid and non-steroid receptor pathways, suggesting that the AIB1 protein might not only affect the cell cycle and cell proliferation, but might also interact with apoptosis genes such as $p 53$ or $B C L 2$, affecting the tumor malignancy phenotype. Esophageal squamous cell carcinoma is one of the most common life-threatening cancers. Its occurrence and development is influenced by multiple genes, and the molecular mechanisms underlying this disorder might provide an important basis for its diagnosis and treatment. Whether AIB1 plays a role in non-hormone-dependent esophageal squamous cell carcinoma is worth investigating (Sun et al., 2008b; Li and Wang, 2011). In order to investigate whether AIB1 plays a role in esophageal squamous cell carcinoma, we collected 60 samples from patients with esophageal squamous cell carcinoma and 20 normal esophageal tissues samples, detected AIB1 expression therein using immunohistochemical methods, and analyzed the correlation parameters with tumor clinical pathology. The results showed that 25 carcinoma specimens (41.6\%) exhibited nuclear AIB1 over-expression, whereas AIB1 expression was weak or absent in the 20 normal esophageal tissue samples $(\mathrm{P}<0.05)$. The expression level of AIB1 in tumor specimens was significantly correlated with clinical stage and lymph metastasis: the expression level of AIB1 in T3-4 stage specimens was higher than that in T1-2 $(\mathrm{P}<0.05)$, and the expression level of AIB1 in tissues with lymph node metastasis was higher than in those without $(\mathrm{P}<0.05)$, whereas expression was not related to gender, age, or tumor grade. These results suggested that AIB1 expression might correlate with the proliferation, invasion, and metastasis of esophageal squamous cell carcinoma. In previous studies, Hmingsen et al. (2003) used immunohistochemistry to detect the expression levels of AIB1 in colorectal cancer and found that high expression of AIB1 was correlated with lymph node metastasis of colorectal cancer; in addition, Sakakura et al. (2000) found that the AIB1 gene was overexpressed in patients with gastric cancer who were prone to extensive lymph node or liver 
metastasis. Furthermore, it was found that the incidence rate was higher in such patients than in those with low expression, and that their prognosis was worse than that of patients without detectable AIB1 expression (Hmingsen et al., 2003), indicating that AIB1 expression was closely related to tumor metastasis and relapse. The above findings are consistent with the conclusions of this study, further supporting that AIB1 might be related to the proliferation, invasion, and metastasis of esophageal squamous cell carcinoma.

Esophageal squamous cell carcinoma is a non-hormone-dependent tumor. Therefore, AIB1 would be predicted to play a role in the proliferation, invasion, and metastasis of esophageal squamous cell carcinoma via a non-steroid hormone receptor pathway. However, the regulatory mechanism of AIB1 with respect to the non-steroid hormone receptor pathway is not yet well understood. Studies have shown that cell cycle, proliferation, and apoptosis genes (such as $p 53$ or BCL2) are related to this regulatory pathway. P53 can activate the DNA damage checkpoint in the G1 phase of the cell cycle as part of the system for monitoring the integrity of the genome. If the genome is damaged, P53 can block DNA replication in order to provide sufficient time for the repair of damaged DNA; failing this, P53 will trigger cellular apoptosis (Lv et al., 2006). Bcl-2, in turn, is the primary target molecule of a variety of apoptosis regulatory mechanisms, serving as an intersection point for multiple pathways and thus occupying an important position in apoptosis. Therefore, the $\mathrm{P} 53$ and $\mathrm{Bcl} 2$ proteins, which are related to the regulatory mechanisms of the AIB1 non-steroid hormone receptor pathway, both play an important role in proliferation and apoptosis. Ki67 is also related to cell division and proliferation. It serves as a positive marker gene for proliferation, is related to the occurrence, development, metastasis, and prognosis of various tumors, and has now become a routine detection index for tumor pathology. Whether Ki67 is related to the AIB1 non-steroidal hormone receptor pathway needs to be further verified. In order to explore the relationship between AIB1 and Ki67, we used immunohistochemistry to detect the expression of AIB1 and Ki67 in esophageal squamous cell carcinoma. Statistical analysis showed that AIB1 and Ki67 expression was moderate correlated $\left(\chi^{2}=30.22, \mathrm{P}=0.000 ; \mathrm{r}=0.579\right)$. This suggested that AIB1 might also be able to interact with Ki67, thereby indirectly regulating proliferation and metastasis, and ultimately affecting tumor stage and lymphatic metastasis or conversely, that Ki67 might be related to the AIB1 non-steroidal hormone receptor pathway, and thus be potentially related to the proliferation, invasion, and metastasis of esophageal squamous cell carcinoma. However, research on the potential role of AIB1 in non-hormone-dependent tumors and their mechanisms has been limited. The potential interaction of AIB1 with other functional proteins and its effect on tumor progression needs to be further studied. In this study, we have drawn preliminary conclusions regarding the expression of AIB1 in esophageal squamous cell carcinoma: that AIB1 plays a specific role in the occurrence and development of esophageal squamous cell carcinoma. In particular, our results have suggested that AIB1 is related to proliferation, invasion, and metastasis, and that in esophageal squamous cell carcinomas with high levels of AIB1 expression, cancer cell proliferation is activated, lending these tumors prone to invasion and metastasis. Furthermore, our findings support that Ki67 is one of the proteins related to the mechanisms of AIB1 function.

\section{Conflicts of interest}

The authors declare no conflict of interest. 


\section{REFERENCES}

Alkner S, Bendahl PO, Grabau D, Lövgren K, et al. (2010). AIB-1 is a predictive factor for tamoxifen response in premenopausal women. Ann. Oncol. 21: 238-244.

Anzick SL, Kononen J, Walker RL, Azorsa DO, et, al. (1997). AIB1, a steroid receptor coactivator amplified in breast and ovarian cancer. Science. 277: 965-968.

Gentile V, Vicini P, Giacomelli L, Cardillo MR, et a1. (2006). Detection of human papillomavirus DNA, p53 and Ki67 expression in penile carcinomas. Int. J. Immunopathol. Pharmacol. 19: 209-215.

Han XY, Chen Y, Hou MM, Zhang J, et al. (2008). Expression of AIB1 protein in epithelial ovarian cancer cells and its impact on apoptosis. Sichuan Da Xue Xue Bao Yi Xue Ban. 39: 619-622, 634.

Hmingsen G, Czerwenka K, Kubista E, Marton E, et al. (2003). Expression of sisterhood receptors and their co-factors in normal, AIB1 is a carcinoma a-specific co-activator. Cancer 78: 193-204.

Jalava P, Kuopio T, Juntti-Patinen L, Kotkansalo T, et al. (2006). Ki67 immunohistochemistry: a valuable marker in prognostication but with a risk of misclassification: proliferation subgroups formed based on Ki67 immunoreactivity and standardized mitotic index. Histopathology 48: 674-682.

Kirkegaard T, McGlynn LM, Campbell FM, Müller S, et al. (2007). Amplified in breast cancer 1 in human epidermal growth factor receptor-positive tumors of tamoxifen-treated breast cancer patients. Clin. Cancer Res. 13: 1405-1411.

Kuang SQ, Liao L, Wang S, Medina D, et al. (2008). Mice lacking the amplified in cancer 1/steroid receptor co activator-3 are resistant to chemical carcinogen-induced mammary tumorigenesis. Cancer Res. 65: 7993-8002.

Lahusen T, Henke RT, Kagan BL, Wellstein A, et al. (2009). The role and regulation of the nuclear receptor coactivator AIB-1 in breast cancer. Breast Cancer Res. Treat. 116: 225-237.

Lee SK, Kim HJ, Kim JW, Lee JW (1999). Steroid receptorcoactivator-1 and its family membersdifferentially regulate transactivation by the tumor suppressor protein p53. Mol Endocrinol. 13: 1924-1933.

Li Z and Wang XC (2011). AIB1 gene in gastrointestinal cancer research in progress. Tai Shan Yi Xue Yuan Xue Bao 28: 394-396.

Lv XD, Wang J, Wang J, Wang LD, et al. (2006). Bcl-2 expression in esophageal and gastric cardia cancers tissue cancer patients and bax and mdm2 and p53 protein. Zhong Hua Zhong Liu Fang Zhi Za Zhi 13: 1285-1288.

Ma G, Ren Y, Wang K and He J (2011). Src-3 has a role in cancer other than as a nuclear receptor coactivator. He J. Int J. Biol Sci. 7: 664-672.

Sakakura C, Hagiwara A, Yasuoka R, Fujita Y, et al. (2000). Amplification and over-expression of the AIB1 nuclear receptor co-activator gene in primary gastric cancer. Int. J. Cancer 89: 217-223.

Sun AM, Li DT, Mu DB, Li H, et al. (2008a). Esophageal squamous cell carcinoma Cyclin D1 and Ki-67 expression and its correlation with prognosis. Zhong Hua Zhong Liu Fang Zhi Za Zhi 15: 276-279.

Sun P, Gao J, Liu YL, Wei LW, et al. (2008b). RNA interference (RNAi)-mediated vascular endothelial growth factor-C (VEGF-C) reduction interferes with lymphangiogenesis and enhances epirubicin sensitivity of breast cancer cells. Mol. Cell. Biochem. 308: 161-168.

Torres-Arzayus MI, Zhao J, Bronson R and Brown M (2010). Estrogen-dependent and estrogen-independent mechanisms contribute to AIB-1-mediated tumor formation. Cancer Res. 70: 4102-4111.

Yamashita H, Takahashi S, Ito Y, Yamashita T, et al. (2009). Predictors of response to exemestane as primary endocrine therapy in estrogen receptor-positive breast cancer. Cancer Sci. 100: 2028-2033.

Zhao W, Zhang Q, Kang X, Jin S, et al. (2009). AIB1 is required for the acquisition of epithelial growth factor receptormediated tamoxifen resistance in breast cancer cells. Biochem. Biophys. Res. Commun. 380: 699-704. 